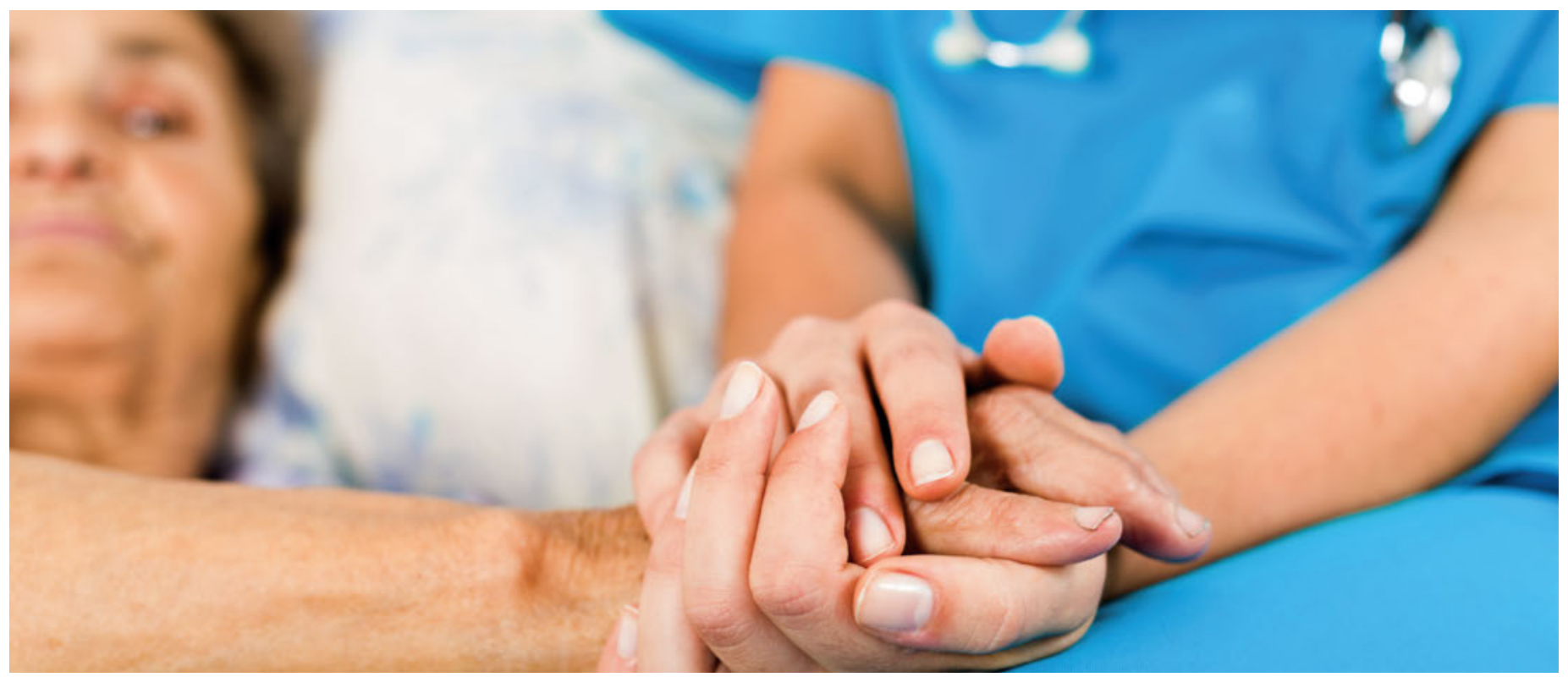

\title{
Betreuung und Behandlung von Menschen mit Demenz
}

\section{Georg Bosshard ${ }^{a}$, Michelle Salathéb}

a PD Dr. med., Klinik für Geriatrie, UniversitätsSpital Zürich (Präsident der verantwortlichen Subkommission), Mitglied FMH

b lic. iur., MAE, stv. Generalsekretärin SAMW

Vgl. www.bag.admin.ch unter «Nationale

Gesundheitsstrategien"

Die neuen medizin-ethischen Richtlinien «Betreuung und Behandlung von Menschen mit Demenz» sind veröffentlicht und ab sofort gedruckt erhältlich. Deren Ausarbeitung erfolgte im Rahmen der Nationalen Demenzstrategie 2014-2019 und in Zusammenarbeit mit der Schweizerischen Gesellschaft für Gerontologie. Die neuen Richtlinien bieten eine praxisbezogene Orientierungshilfe zu ethischen Fragen und Konfliktsituationen in der Betreuung und Behandlung von Menschen mit Demenz.

Das Thema bewegt: In der Vernehmlassung von Juni bis August 2017 ist der Richtlinienentwurf auf grosses Interesse gestossen, über 80 Institutionen, Organisationen und Einzelpersonen haben dazu Stellung genommen. Die zuständige Subkommission unter dem Vorsitz von PD Dr. Georg Bosshard, Zürich, hat die teilweise sehr ausführlichen Rückmeldungen diskutiert und nach Möglichkeit in den Richtlinientext integriert resp. entsprechende Modifikationen vorgenommen. Insgesamt waren die Rückmeldungen sehr positiv. Hervorgehoben wurden insbesondere Ausgewogen- heit, hohe Praxisrelevanz und gute Verständlichkeit des Richtlinienentwurfes.

Vereinzelt wurde kritisiert, dass der Text zu «medizinlastig» sei und zu sehr einen pathologisierenden statt einen verstehenden Ansatz vertrete. In diesem Zusammenhang möchten wir darauf hinweisen, dass die SAMW-Richtlinien sich in erster Linie an medizinische Fachpersonen wenden und dass eine Ergänzung der Richtlinien für nicht-medizinische Berufe unter Federführung der Schweizerischen Gesellschaft für Gerontologie vorgesehen ist. 
Mit Blick auf spezifische Themen der Richtlinien waren es nicht unerwartet die "Zwangsmassnahmen" und die "Fürsorgerische Unterbringung», die in der Vernehmlassung zu zahlreichen Rückmeldungen geführt haben. Die Forderung, dass bei bewegungseinschrän-

\section{In der Vernehmlassung von Juni bis August 2017 ist der Richtlinienentwurf auf grosses Interesse gestossen; über 80 Stellungnahmen wurden eingereicht.}

kenden Massnahmen generell die gesetzliche Vertretungsperson informiert werden und diese generell zu medizinischen Zwangsmassnahmen (Verabreichung von Medikamenten) zustimmen muss, wurde insbesondere von Ärztinnen und Ärzten in Spitälern als nicht immer praktikabel beurteilt. Dieser Kritik wurde in der definitiven Version durch folgenden Zusatz Rechnung getragen: «Vorbehalten bleiben Notfallsituationen und absehbar vorübergehende Krisensituationen, wie sie insbesondere bei hospitalisierten Patienten mit Demenz vorkommen können.»

Ausgesprochen widersprüchlich waren die Rückmeldungen zur Empfehlung des Beizugs der Kindes- und Erwachsenenschutzbehörde (KESB), wenn ein demenzkranker Mensch mit der Unterbringung in einer Institution nicht einverstanden ist. Einerseits wurde befürchtet, dass - gestützt auf die SAMW-Richtlinien Platzierungen gegen den Willen einer Person ohne Involvierung der KESB und ohne formales, rekursfähiges Verfahren erfolgen könnten. Andererseits wurde darauf hingewiesen, dass Menschen mit Demenz wegen fehlender Krankheitseinsicht nur selten mit einer Institutionalisierung einverstanden seien, so dass gestützt auf die Richtlinien dann praktisch immer die KESB einbezogen werden müsste. Dies wird als nicht sinnvoll und unpraktikabel beurteilt. Die definitive Version der Richtlinien enthält deshalb gegenüber dem Vernehmlassungsentwurf Präzisierungen, damit auch solch kontrovers diskutierten Stellen einen klaren Orientierungsrahmen für die Praxis bieten.

Abschliessend wurde in vielen Stellungnahmen betont, dass die erfolgreiche Umsetzung der Richtlinien hohe Anforderungen an das Betreuungspersonal stellt und dass genügend strukturelle und personelle Ressourcen bereitgestellt werden müssen. Dies ist als Aufforderung an die Praxis zu verstehen. Die Richtlinien wenden sich an Ärztinnen und Ärzte, Pflegefachleute und weitere therapeutisch tätige Fachpersonen.

\section{Die Richtlinien wenden sich an Ärztinnen und Ärzte, Pflegefachleute und weitere therapeu- tisch tätige Fachpersonen.}

Themen auf übergeordneter Ebene - etwa der Auf- und Ausbau bedarfsgerechter Versorgungsangebote entlang der gesamten Versorgungskette - werden in den Richtlinien nicht behandelt. Diese Themen Themen auf übergeordneter Ebene - z.B. der Auf- und Ausbau bedarfsgerechter Versorgungsangebote entlang der Versorgungskette - nicht in den Richtlinien behandelt. Diese Themen, die in weiteren Handlungsfeldern der Nationalen Demenzstrategie vertieft werden, sind jedoch Voraussetzung für die Umsetzbarkeit der Richtlinien.

\section{Bildnachweis}

(C) Lighthunter | Dreamstime.com

\section{Hinweis}

Die medizin-ethischen Richtlinien der SAMW stehen deutsch, französisch, italienisch und englisch als Download zur Verfügung. Gedruckte Exemplare (d/f) sind kostenlos erhältlich und können online auch in grösseren Mengen bestellt werden: samw.ch/richtlinien. 